\title{
Honeycomb Arrays
}

\author{
Simon R. Blackburn* Anastasia Panoui* \\ Maura B. Paterson ${ }^{\dagger} \quad$ Douglas R. Stinson ${ }^{\ddagger}$
}

Submitted: Nov 12, 2009; Accepted: Nov 17, 2010; Published: Dec 10, 2010

Mathematics Subject Classification: 05B30

\begin{abstract}
A honeycomb array is an analogue of a Costas array in the hexagonal grid; they were first studied by Golomb and Taylor in 1984. A recent result of Blackburn, Etzion, Martin and Paterson has shown that (in contrast to the situation for Costas arrays) there are only finitely many examples of honeycomb arrays, though their bound on the maximal size of a honeycomb array is too large to permit an exhaustive search over all possibilities.

The present paper contains a theorem that significantly limits the number of possibilities for a honeycomb array (in particular, the theorem implies that the number of dots in a honeycomb array must be odd). Computer searches for honeycomb arrays are summarised, and two new examples of honeycomb arrays with 15 dots are given.
\end{abstract}

\section{Introduction}

Honeycomb arrays were introduced by Golomb and Taylor [8] in 1984, as a hexagonal analogue of Costas arrays. Examples of honeycomb arrays are given in Figures 7 to 11 below. A honeycomb array is a collection of $n$ dots in a hexagonal array with two properties:

- (The hexagonal permutation property) There are three natural directions in a hexagonal grid (see Figure 1). Considering 'rows' in each of these three directions, the dots occupy $n$ consecutive rows, with exactly one dot in each row.

- (The distinct differences property) The $n(n-1)$ vector differences between pairs of distinct dots are all different.

*Department of Mathematics, Royal Holloway, University of London, Egham, Surrey TW20 0EX, United Kingdom. \{s.blackburn, a.panoui\}@rhul.ac.uk

${ }^{\dagger}$ Department of Economics, Mathematics and Statistics, Birkbeck, University of London, Malet Street, London WC1E 7HX, United Kingdom. m.paterson@bbk.ac.uk

${ }_{\ddagger}^{\ddagger}$ David R. Cheriton School of Computer Science, University of Waterloo, Waterloo Ontario, N2L 3G1, Canada. dstinson@uwaterloo.ca 


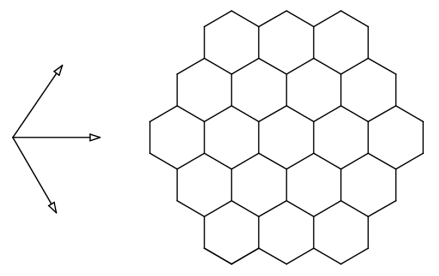

Figure 1: A Lee sphere, and three natural directions

Golomb and Taylor found 10 examples of honeycomb arrays (up to symmetry), and conjectured that infinite families of honeycomb arrays exist. Blackburn, Etzion, Martin and Paterson [5] recently disproved this conjecture: there are only a finite number of honeycomb arrays. Unfortunately, the bound on the maximal size of a honeycomb array that Blackburn et al. provide is far too large to enable an exhaustive computer search over all open cases. In this paper, we prove a theorem that significantly limits the possibilities for a honeycomb array with $n$ dots. (In particular, we show that $n$ must be odd.) We report on our computer searches for honeycomb arrays, and give two previously unknown examples with 15 dots.

We now introduce a little more notation, so that we can state the main result of our paper more precisely.

We say that a collection of dots in the hexagonal grid is a hexagonal permutation if it satisfies the hexagonal permutation property. A collection of dots is a distinct difference configuration if it satisfies the distinct difference property. So a honeycomb array is a hexagonal permutation that is a distinct difference configuration.

We say that hexagons are adjacent if they share an edge, and we say that two hexagons $A$ and $B$ are at distance $d$ if the shortest path from $A$ to $B$ (travelling along adjacent hexagons) has length $d$. A Lee sphere of radius $r$ is a region of the hexagonal grid consisting of all hexagons at distance $r$ or less from a fixed hexagon (the centre of the sphere). The region in Figure 1 is a Lee sphere of radius 2. Note that a Lee sphere of radius $r$ intersects exactly $2 r+1$ rows in each of the three natural directions in the grid. A honeycomb array of radius $r$ is a honeycomb array with $2 r+1$ dots contained in a Lee sphere of radius $r$.

There are many other natural regions of the hexagonal grid that have the property that they intersect $n$ rows in each direction. One example, the tricentred Lee sphere of radius $r$, is shown in Figure 2: it is the union of three Lee spheres of radius $r$ with pairwise adjacent centres, and intersects exactly $2 r+2$ rows in any direction.

Does there exist a honeycomb array with $2 r+2$ dots contained in a tricentred Lee sphere of radius $r$ ? Golomb and Taylor did not find any such examples: they commented [8, Page 1156] that all known examples of honeycomb arrays with $n$ dots were in fact honeycomb arrays of radius $r$, but stated "we have not proved that this must always be the case". We prove the following: 


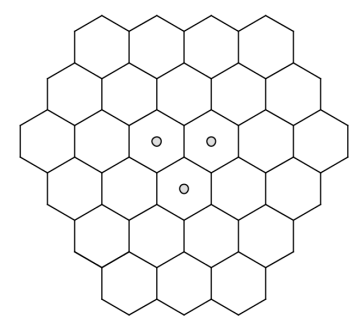

Figure 2: A tricentred Lee sphere

Theorem 1 Let $n$ be an integer, and suppose there exists a hexagonal permutation $\pi$ with $n$ dots. Then $n$ is odd, and the dots of $\pi$ are contained in a Lee sphere of radius $(n-1) / 2$.

Since any honeycomb array is a hexagonal permutation, the following result follows immediately from Theorem 1:

Corollary 2 Any honeycomb array is a honeycomb array of radius $r$ for some integer $r$. In particular, a honeycomb array must consist of an odd number of dots.

So if we are looking for honeycomb arrays, we may restrict ourselves to searching for honeycomb arrays of radius $r$.

The structure of the remainder of the paper is as follows. In Section 2, we state the results on the hexagonal grid that we need. In Section 3, we remind the reader of the notion of a brook, or bee-rook, and state a theorem on the maximum number of nonattacking brooks on a triangular board. In Section 4 we prove Theorem 1, we summarise our computer searches for honeycomb arrays, and we provide a list of all known arrays. This last section also contains a conjecture, and some suggestions for further work.

\section{The hexagonal grid}

Because the hexagonal grid might be difficult to visualise, we use an equivalent representation in the square grid (see Figure 3). In this representation, we define each square to be adjacent to the four squares it shares an edge with, and the squares sharing its 'North-East' and 'South-West' corner vertices. The map $\xi$ in Figure 3 distorts the centres of the hexagons in the hexagonal grid to the centres of the squares in the square grid. The three types of rows in the hexagonal grid become the rows, the columns and the diagonals that run from North-East to South-West. For brevity, we define a standard diagonal to mean a diagonal that runs North-East to South-West.

For non-negative integers $n$ and $i$ such that $0 \leq i \leq n-1$, define the region $S_{i}(n)$ of the square grid as in Figure 4 . Note that $S_{i}(n)$ and $S_{n-1-i}(n)$ are essentially the same region: one is obtained from the other by a reflection in a standard diagonal. The regions $\xi^{-1}\left(S_{i}(n)\right)$ are important in the hexagonal grid, as they are the maximal anticodes of diameter $n-1$; see Blackburn et al. [5, Theorem 5]. Note that the region $\xi^{-1}\left(S_{r}(2 r+1)\right)$ is a Lee sphere of radius $r$. Regions of the form $\xi^{-1}\left(S_{r}(2 r+2)\right)$ or $\xi^{-1}\left(S_{r+1}(2 r+2)\right)$ are tricentred Lee spheres of radius $r$. Also note that the regions $S_{i}(n)$ as $i$ varies are precisely 

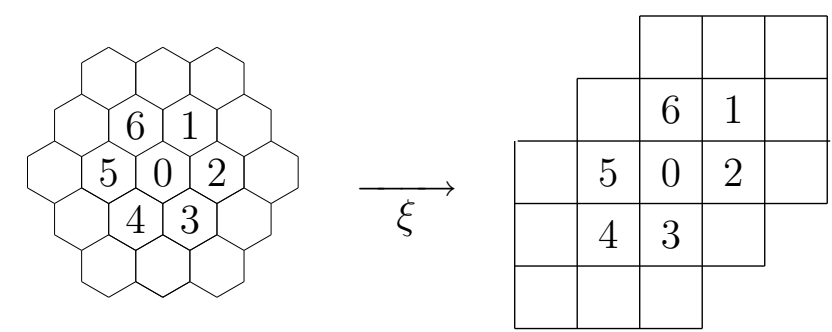

Figure 3: From the hexagonal to the square grid

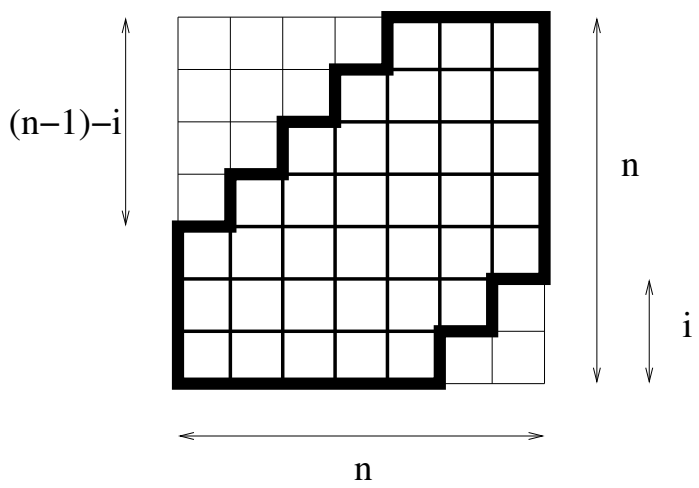

Figure 4: The region $S_{i}(n)$

the possible intersections of an $n \times n$ square region with $n$ adjacent standard diagonals, where each diagonal intersects the $n \times n$ square non-trivially.

In the lemma below, by a 'region of the form $X$ ', we mean a region that is a translation of $X$ in the square grid.

Lemma 3 Let $\pi$ be a hexagonal permutation with $n$ dots, and let $\xi(\pi)$ be the image of $\pi$ in the square grid. Then the dots in $\xi(\pi)$ are all contained in a region of the form $S_{i}(n)$ for some $i$ in the range $0 \leq i \leq n-1$.

Proof: Let $R$ be the set of squares that share a row with a dot of $\xi(\pi)$. Similarly, let $C$ and $D$ be the sets squares sharing respectively a column or a standard diagonal with a dot of $\xi(\pi)$. The dots in $\xi(\pi)$ are contained in $R \cap C \cap D$.

Since $\pi$ is a hexagonal permutation, $R$ consists of $n$ adjacent rows and $C$ consists of $n$ adjacent columns. Hence $R \cap C$ is an $n \times n$ square region. (Since there is exactly one dot in each row and column of the square $R \cap C$, the dots in $\xi(\pi)$ correspond to a permutation; this justifies the terminology 'hexagonal permutation'.)

Now, $D$ consists of $n$ adjacent standard diagonals; each diagonal contains a dot in $\xi(\pi)$, and so each diagonal intersects $R \cap C$ non-trivially. Hence $R \cap C \cap D$ is a region of the form $S_{i}(n)$, as required. 


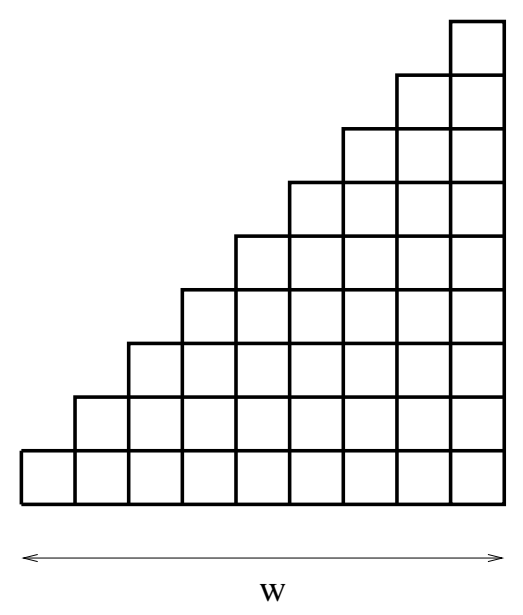

Figure 5: A triangular board of width $w$

\section{Brooks on a triangular board}

A brook is a chess piece in the square grid that moves like a rook plus half a bishop: it can move any distance along a row, a column or a standard (North-East to SouthWest) diagonal. Brooks were first studied by Bennett and Potts [4], who pointed out connections to constant-sum arrays and hexagonal lattices. A set of brooks in a square grid is non-attacking if no two brooks lie in a row, a column or a standard diagonal.

Under the correspondence $\xi$ between the square and hexagonal grids mentioned in the previous section, brooks in the square grid correspond to bee-rooks in the hexagonal grid: pieces that can move any distance along any row, where a row can go in each of the three natural directions. A set of bee-rooks is therefore non-attacking if no two bee-rooks lie in the same row of the hexagonal grid. In particular, bee-rooks placed on the dots in a hexagonal permutation $\pi$ are non-attacking, and so the corresponding set $\xi(\pi)$ of brooks in the square grid is non-attacking.

A triangular board of width $w$ is the region $S_{0}(w)$ in the square grid depicted in Figure 5. Let $b(w)$ be the maximum number of non-attacking brooks that can be placed in the triangular board of width $w$. The following theorem is proved by Nivasch and Lev [10] and in Vaderlind, Guy and Larson [12, P252 and R252]:

Theorem 4 For any positive integer $w, b(w)=\lfloor(2 w+1) / 3\rfloor$.

Three of the present authors have found an alternative proof for this theorem, using linear programming techniques [6]. See Bell and Stevens [3] for a survey of similar combinatorial problems.

\section{Honeycomb arrays}

We begin this section with a proof of Theorem 1. We then describe our searches for honeycomb arrays. We end the section by describing some avenues for further work. 


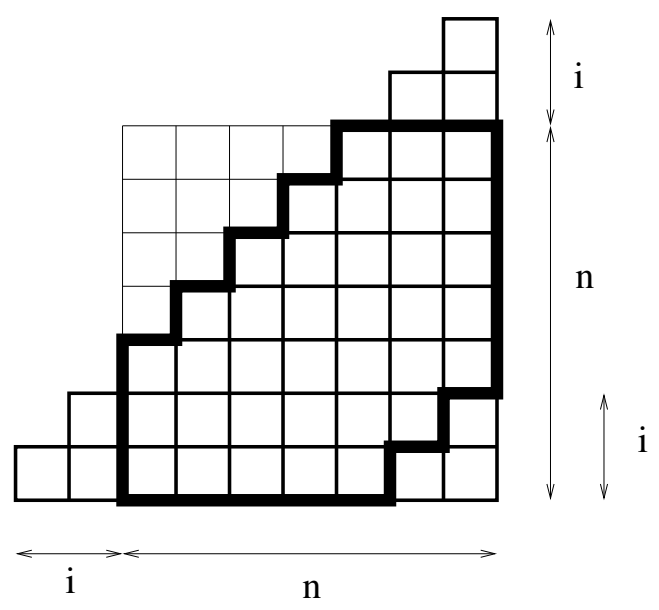

Figure 6: A triangular board covering $\pi$

Proof of Theorem 1: Let $\pi$ be a hexagonal permutation with $n$ dots. By Lemma 3, the dots of $\xi(\pi)$ are contained in a region of the form $S_{i}(n)$ where $0 \leq i \leq n-1$. When $i=(n-1) / 2$ (so $n$ is odd and $\xi^{-1}\left(S_{i}(n)\right)$ is a Lee sphere) the theorem follows. Suppose, for a contradiction, that $i \neq(n-1) / 2$.

By reflecting $\pi$ in a horizontal row in the hexagonal grid, we produce a hexagonal permutation $\pi^{\prime}$ such that $\xi\left(\pi^{\prime}\right)$ is contained in a region of the form $S_{(n-1)-i}(n)$. By replacing $\pi$ by $\pi^{\prime}$ if necessary, we may assume that $i<(n-1) / 2$.

Consider the triangular board of width $n+i$ in Figure 6 containing $S_{i}(n)$. Since no two dots in $\xi(\pi)$ lie in the same row, column or standard diagonal, the dots in $\xi(\pi)$ correspond to $n$ non-attacking brooks in this triangular board. But this contradicts Theorem 4 , since

$$
\frac{2(n+i)+1}{3}<\frac{2 n+(n-1)+1}{3}=n .
$$

This contradiction completes the proof of the theorem.

Theorem 1 tells us that the only honeycomb arrays are those of radius $r$ for some non-negative integer $r$. A result of Blackburn et al [5, Corollary 12] shows that $r \leq 643$. We now report on our computer searches for examples of honeycomb arrays. The known honeycomb arrays are drawn in Figures 7, 8, 9, 10 and 11. This list includes two new examples not known to Golomb and Taylor [8], namely the second and third examples of radius 7 ; we found these examples as follows.

A Costas array is a set of $n$ dots in an $n \times n$ region of the square grid, with the distinct difference property and such that every row and column of the array contains exactly one dot. Golomb and Taylor observed that some Costas arrays produce honeycomb arrays, by mapping the dots in the Costas array into the hexagonal grid using the map $\xi^{-1}$ given by Figure 3. Indeed, it is not difficult to see that all honeycomb arrays must arise in this way. We searched for honeycomb arrays by taking each known Costas array with 200 or fewer dots, and checking whether the array gives rise to a honeycomb array. For our search, we made use of a database of all known Costas arrays with 200 or fewer dots that has been made available by James K. Beard [1]. This list is known to be complete 

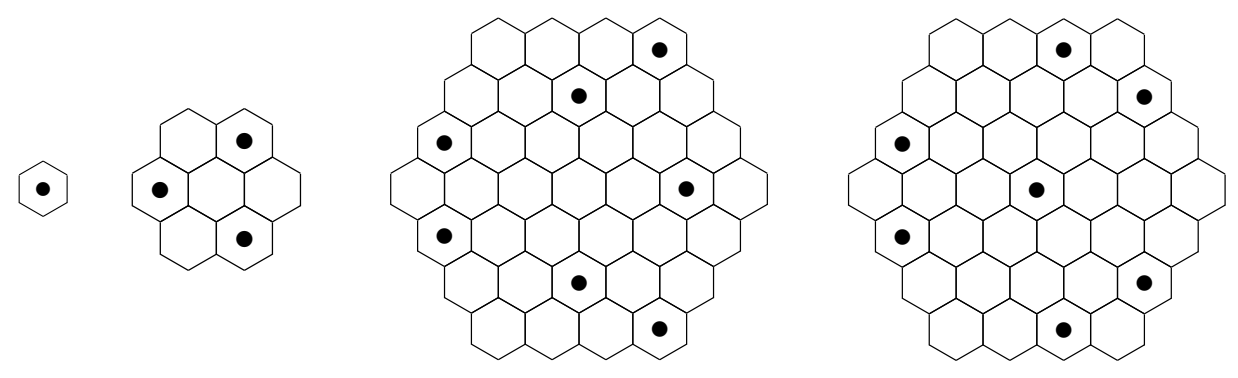

Figure 7: Honeycomb arrays of radius 0,1 and 3
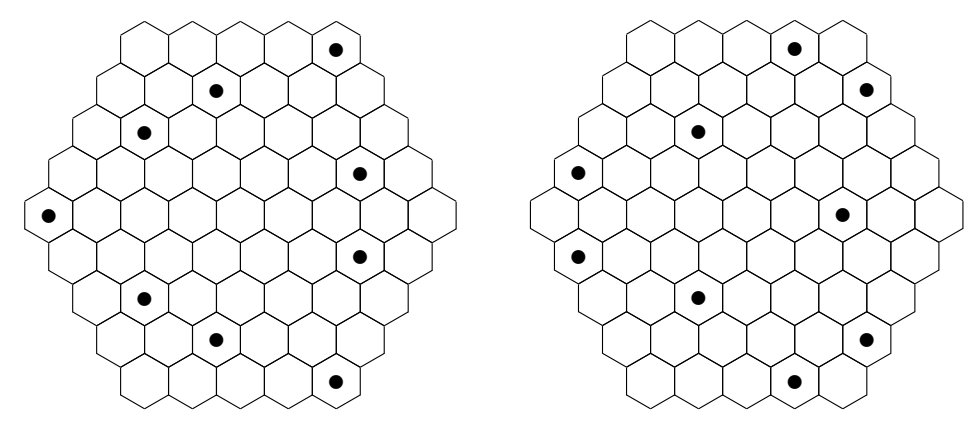

Figure 8: Honeycomb arrays of radius 4

for Costas arrays with 27 or fewer dots; see Drakakis et al. [7] for details. So our list of honeycomb arrays of radius 13 or less is complete.

It is a remarkable fact that all known honeycomb arrays possess a non-trivial symmetry (a horizontal reflection as we have drawn them). Indeed, apart from a single example of radius 3 (the first radius 3 example in Figure 7) all known honeycomb arrays possess a symmetry group of order 6: the group generated by the reflections along the three lines through opposite 'corners' of the hexagonal sphere. We implemented an exhaustive search for honeycomb arrays with $r \leq 31$ having this 6-fold symmetry: we found no new examples. We also checked all constructions of honeycomb arrays from Costas arrays in Golomb and Taylor [8] (whether symmetrical or not) for $r \leq 325$, and again we found no new examples.
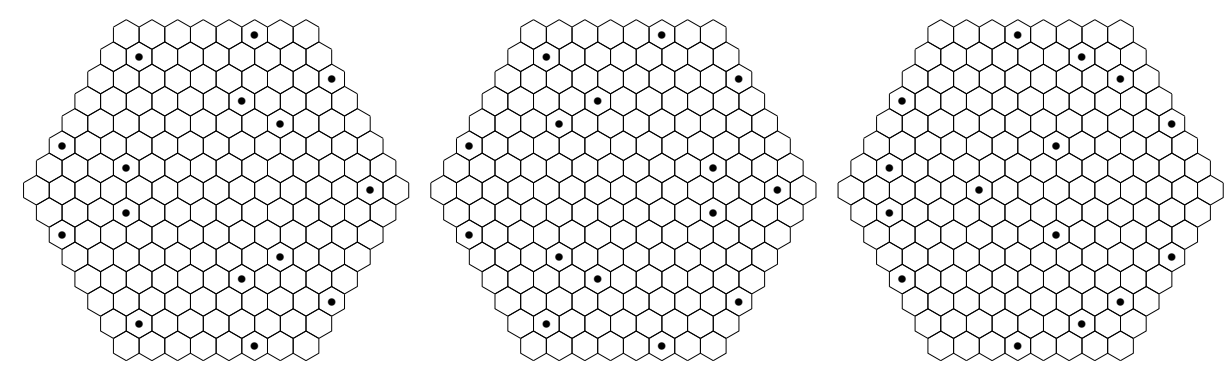

Figure 9: Honeycomb arrays of radius 7 

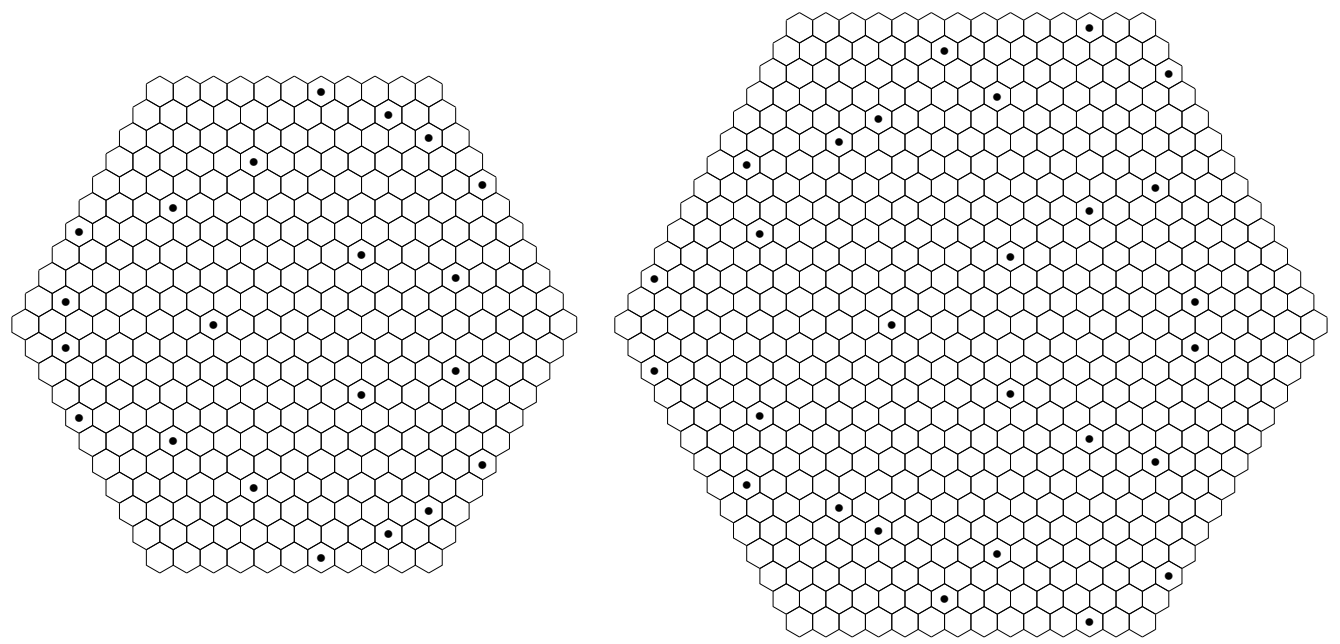

Figure 10: Honeycomb arrays of radius 10 and 13

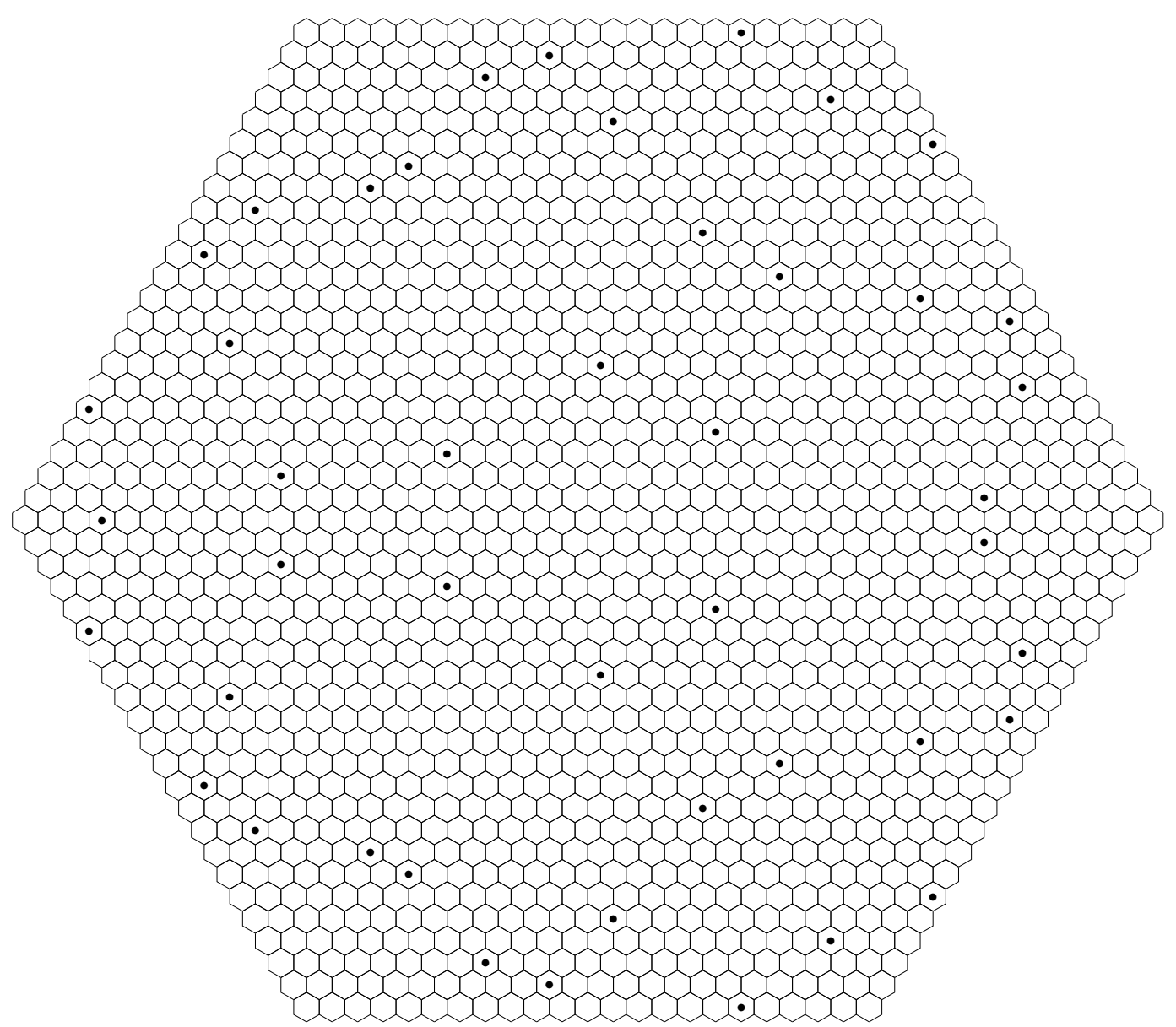

Figure 11: A honeycomb array of radius 22 
After these searches, we feel that we can make the following conjecture:

Conjecture 1 The list of known honeycomb arrays is complete. So there are are exactly 12 honeycomb arrays, up to symmetry.

Theorem 1 shows that hexagonal permutations are always contained in some Lee sphere. But such permutations have been prevously studied in several contexts: Bennett and Potts [4] study them as non-attacking configurations of bee-rooks and as the number of zero-sum arrays; Kotzig and Laufer [9] study them as the number of $\sigma$-permutations; Bebeacua, Mansour, Postnikov and Severini [2] study them as X-rays of permutations with maximum degeneracy. Let $h_{n}$ be the number of hexagonal permutations with $2 n-1$ dots. The On-Line Encyclopedia of Integer Sequences [11, Sequence A002047] quotes a computation due to Alex Fink that computes the first few terms of the sequence $h_{n}$ :

\begin{tabular}{c|cccccccccc}
$n$ & 1 & 2 & 3 & 4 & 5 & 6 & 7 & 8 & 9 & 10 \\
\hline$h_{n}$ & 1 & 2 & 6 & 28 & 244 & 2544 & 35600 & 659632 & 15106128 & 425802176
\end{tabular}

Kotzig and Laufer ask: How big can $h_{n}$ be? It seems that the sequence grows faster than exponentially with $n$. We ask a more precise question: Is it true that $\left(\log h_{n}\right) / n \log n$ tends to a constant as $n \rightarrow \infty$ ?

Acknowledgements The authors would like to thank Tuvi Etzion for discussions, funded by a Royal Society International Travel Grant, which inspired this line of research. Part of this work was completed under EPSRC Grant EP/D053285/1. Doug Stinson's research was supported by NSERC discovery grant 203114-06.

\section{References}

[1] James K. Beard, http://jameskbeard.com/jameskbeard/

[2] Cecilia Bebeacua, Toufik Mansour, Alex Postnikov and Simone Severini, 'On the X-rays of permutations', Elec. Notes Disc. Math. 20 (2005) 193-203.

[3] Jordan Bell and Brett Stevens, 'A survey of known results and research areas for n-queens', Discrete Math. 309 (2009) 1-31.

[4] B.T. Bennett and R.B. Potts, 'Arrays and brooks', J. Australian Math. Soc. 7 (1967) 23-31.

[5] Simon R. Blackburn, Tuvi Etzion, Keith M. Martin and Maura B. Paterson, 'Twodimensional patterns with distinct differences - Constructions, bounds and maximal anticodes', IEEE Trans. Inform. Theory 56 (2010) 1216-1229.

[6] Simon R. Blackburn, Maura B. Paterson and Douglas R. Stinson, 'Putting dots in triangles', J. Combin. Math. Combin. Comput., to appear. http://arxiv.org/abs/ 0910.4325 . 
[7] Konstantinos Drakakis, Scott Rickard, James K Beard, Rodrigo Caballero, Francesco Iorio, Gareth O'Brien and John Walsh, 'Results of the enumeration of Costas arrays of order 27', IEEE Trans. Inform. Theory 54 (2008) 4684-4687.

[8] Solomon W. Golomb and Herbert Taylor, 'Constructions and properties of Costas arrays', Proc. IEEE 72 (1984) 1143-1163.

[9] A. Kotzig and P.J. Laufer, 'When are permutations additive?', American Math. Monthly 85 (1978), 364-365.

[10] Gabriel Nivasch and Eyal Lev, 'Nonattacking queens on a triangle', Math. Magazine 78 (2005) 399-403.

[11] N.J.A. Sloane (Ed.), The On-Line Encyclopedia of Integer Sequences, http://www. research.att. com $/ \sim$ njas/sequences/.

[12] Paul Vaderlind, Richard Guy and Loren Larson, The Inquisitive Problem Solver (Mathematical Assoc. of America, Washington, 2002). 